\title{
Review of: "Functional and molecular characterization of a non-human primate model of autism spectrum disorder shows similarity with the human disease"
}

\author{
Mamiko Koshiba ${ }^{1}$ \\ 1 Yamaguchi University
}

Potential competing interests: The author(s) declared that no potential competing interests exist.

This manuscript describes a praiseworthy comparative study about the function and the set of gene relation of human brain development in marmoset.

I think the predictable genetic functional mechanism map, Supplementary Figure 7, seems suitable to be presented in the main text in Discussion chapter.

If I wished an additional request in only behavioral index, it might be better that isolation vocal call entropy (Figure 3) should be evaluated any correlation with gene modulation because the authors show here as a developmental function of brain.

This paper is generally worthy of acceptance, if possible, with considering these points above. 